\title{
Value of dedicated research time for IMGs in obtaining surgical residency training positions: A 10-year review of applicants from a medical college in Pakistan
}

\author{
Mohammad Raheel Jajja \\ Emory University, Atlanta \\ Marvi Tariq \\ Aga Khan University \\ Salila Shoaib Hashmi \\ Emory University, Atlanta \\ Thomas F. Dodson \\ Emory University, Atlanta, Georgia \\ Rashida Ahmed \\ Aga Khan University, rashida.ahmed@aku.edu
}

Follow this and additional works at: https://ecommons.aku.edu/pakistan_fhs_mc_pathol_microbiol

Part of the Microbiology Commons, and the Pathology Commons

\section{Recommended Citation}

Jajja, M. R., Tariq, M., Hashmi, S. S., Dodson, T. F., Ahmed, R. (2019). Value of dedicated research time for IMGs in obtaining surgical residency training positions: A 10-year review of applicants from a medical college in Pakistan. Journal of Surgical Education, 76(1), 43-49.

Available at: https://ecommons.aku.edu/pakistan_fhs_mc_pathol_microbiol/1122 


\title{
Value of Dedicated Research Time for IMGs in Obtaining Surgical Residency Training Positions: A 10-Year Review of Applicants from a Medical College in Pakistan
}

\author{
Mohammad Raheel Jaija, MBBS, * ${ }^{\dagger}$ Marvi Tariq, MBBS, ${ }^{\ddagger}$ Salila Shoaib Hashmi, MBBS, * \\ Thomas F Dodson, MD, FACS, ${ }^{*}$ and Rashida Ahmed, MBBS, MHPE, FCPS ${ }^{\$}$
}

\begin{abstract}
*Department of Surgery, Emory University, Atlanta, GA; ${ }^{\dagger}$ Winship Cancer Institute, Emory University, Atlanta, GA; ¥Medical College, Aga Khan University, Karachi, Pakistan; and \$Department of Pathology and Laboratory Medicine, Aga Khan University, Karachi, Pakistan
\end{abstract}

OBJECTIVE: International Medical Graduates (IMGs) secured greater than $10 \%$ of all general surgery (GS) residency positions in the US during the past decade. The Match process remains competitive, with a significant number of IMGs performing dedicated research before residency application. The impact of such research remains largely unknown. We aimed to provide an objective analysis of the impact of dedicated research time on obtaining a categorical GS residency position.

DESIGN: Data for National Resident Matching Program Match results from 2008-2017 was compiled from annual Match lists of the Aga Khan University, Medical College (Karachi, Pakistan). Medical graduates provided this information voluntarily each year. Data was exported to Microsoft Excel and used for descriptive and statistical analysis using SPSS. Candidates were divided into quasiexperimental groups based on their preference for direct application (no-research group, $n=64$ ) or research prior to Match (research group, $\mathrm{n}=20$ ).

RESULTS: A total of 84 IMG applicants matched into GS residency positions in the US within the past decade. Amongst these, 18 matched directly into categorical

\footnotetext{
Correspondence: Inquiries to Mohammad Raheel Jajja, Winship Cancer Institute, C-4038, Bench 25, 1365-C Clifton Road, Northeast Atlanta, GA 30322; e-mail: raheeljajja@gmail.com

Declarations of Interest: None Competing Interests: None.

${ }^{*}{ }^{*}$ Informed Consent: All data published is from publicly available sources, AKUNET (previously AKUTopica) forums as well as on the AKU alumni website. All data presented here is deidentified.

${ }^{k-k}{ }^{-k}$ Funding: This research did not receive any specific grant from funding agencies in the public, commercial, or not-for-profit sectors.
}

positions while 66 applicants secured preliminary spots. A total of 37 (56\%) preliminary candidates eventually secured categorical GS residency positions. Research group applicants had an overall $85 \%(\mathrm{n}=17)$ success rate of obtaining a categorical position, while no-research group had a $59 \%(n=39)$ success rate (chi-square test, $\mathrm{p}=0.04)$. Success rate was $69 \%(\mathrm{n}=38)$ for male applicants and 57\% $(n=17)$ for female applicants. Median time to a categorical position was 4 years (2-6) for the research group and 3 years (1-6) for the no-research group.

CONCLUSIONS: Our quasi-experimental study demonstrated a higher success rate for Aga Khan University, Medical College applicants with significant research background in the US, compared to those who did not. Better social integration, enhanced mentorship available during research, overcoming of cultural and linguistic barriers and a perception as better qualified candidate can be some factors contributing to higher success rates. (J Surg Ed 000:1-7. (C) 2018 Association of Program Directors in Surgery. Published by Elsevier Inc. All rights reserved.)

ACGME COMPETENCIES: Systems-Based Practice, Professionalism

KEY WORDS: Surgical Residency, International Medical Graduates (IMGs), Residency Applications, Selection Criteria, Research

\section{INTRODUCTION}

International medical graduates (IMGs) are an integral component of the United States physician workforce. ${ }^{1}$ 
Currently, 1 in 4 physicians practicing in the United States received their initial medical school training in a foreign country, representing an increase of $160 \%$ from $1973 .^{2}$ Overall, $27 \%$ of current residents and fellows in specialty programs in the United States have graduated from medical schools abroad. ${ }^{3,4}$ At present, general surgery residency positions remain in the top 5 desired residency specialties for IMGs. ${ }^{5}$ In 1995, Whitcomb et al. ${ }^{6}$ reported that $12 \%$ of all general surgery residency programs in the United States were "IMG dependent." A study by Horvath et al. ${ }^{7}$ showed that there has been more than a $10 \%$ increase in the number of positions filled by IMGs in both preliminary and categorical tracks since 2012. This contrasts with the data for all other PGY1 specialties, where the number of non-US IMG's has declined over this period. ${ }^{8}$ According to National Resident Matching Program (NRMP) data, IMGs have constituted more than $10 \%$ of all preliminary and categorical residency spots filled in the US from 2008-2017.?

The IMG workforce remains an important resource for provision of surgical care in the US. ${ }^{10}$ Most IMGs have trained in medical schools in India (23\%), Caribbean (17\%), Philippines (6\%), and Pakistan (6\%). ${ }^{11}$ IMGs constitute $10 \%$ of all academic surgical faculty in the US, but they are less than $6 \%$ of the resident work force, meaning a higher proportion of IMGs enter academic workforce. IMGs have been documented to more frequently provide care to underserved and rural populations compared with US graduates, offsetting a potentially significant gap in surgical care. ${ }^{13}$ Studies have shown the performance of IMGs in surgical residency programs to be comparable with their US counterparts, with similar scores on the annual American board of surgery in-training examinations. ${ }^{14}$

To demonstrate their commitment and interest in the field of surgery, many IMGs choose to utilize a year or more doing dedicated research in the US before entering the Match cycle. Along with monetary support, these positions provide them with mentorship and networking opportunities that can prove valuable during the Match. ${ }^{15}$ However, most IMGs end up matching in preliminary positions, ${ }^{16}$ with a select few eventually managing to secure categorical spots. ${ }^{3}$ Perceived differences in medical knowledge, clinical and interpersonal skills, ${ }^{17,18}$ potential concerns regarding program reputation, ${ }^{19}$ and a documented higher failure rate in board examinations ${ }^{10}$ in the past all serve to make it harder for IMGs to secure general surgery residency positions.

We have been following self-reported Match results of general surgery applicants to the US, from the Aga Khan University (AKU), Medical College (Karachi, Pakistan) for the last 10 years. AKU's medical school has historically had at least $80 \%$ of its graduating students match annually via NRMP to residency positions in the US since 1988. AKU has a very selective merit based admission policy to its medical school, typically accepting 3\%-5\% of applicants. The medical school has 90-100 graduates annually. While the medical school class has an ethnically and socially diverse makeup, the academic records are very homogenous with a typical student being a high achiever on standardized tests (General Certificate of Education, International Baccalaureate, Scholastic Aptitude Test, and Higher Education Commission of Pakistan exams). While reviewing the 10-year data from these compiled Match lists we identified a clear dichotomy in the general surgery applicant pool from this medical school: (1) a proportion of medical graduates pursued dedicated research, followed by application for surgical residency and (2) the remaining applying directly for surgical residencies. This set up the basis for what would be considered a natural experiment (quasi-experimental study $)^{20,21}$ for us to analyze.

\section{MATERIALS AND METHODS}

This study used data from 2008-2017 collated from information provided voluntarily by medical graduates at the AKU, Medical College yearly at the end of each Match cycle. This data is compiled into predefined "Match Lists" at the end of each Match season by AKU alumni. These lists are comprehensively vetted before being disseminated to alumni and current students. A record of all these lists can be publicly found on AKUNET (previously AKUTopica) forums as well as on the AKU alumni website. $^{22}$ In addition, data from these lists was vetted by directly contacting the included individuals where possible. Please note that while AKU does also have medical school campuses in East Africa (Nairobi, Kenya, and Dar es Salaam, Tanzania), where postgraduate courses are offered, these Match lists were restricted to include only graduates of undergraduate programs from the Karachi, Pakistan campus.

The matched applicants were split into our quasiexperimental groups (1) those who spent dedicated time ( $>6$ months) doing clinical or lab-based research in the United States prior to applying for the Match (research group, $\mathrm{n}=20$ ); (2) those who applied directly for the Match (no-research group, $n=64$ ).

Data analysis was performed using SPSS version 24 (IBM Corp.). Comparisons of proportions between 2 groups were made utilizing chi-square or Fisher's exact test, where applicable. Continuous data was analyzed using 2-sample t-tests for means or Mann-Whitney tests for medians where appropriate. Data is presented as mean values with standard deviations, medians with ranges, or as counts. 


\section{RESULTS}

Over the 10-year study period, a total of 84 applicants from AKU matched into general surgery preliminary and categorical residency positions in the United States, 54 applicants were male, and 30 were female. Out of these, 18 (21.4\%) matched directly into categorical positions, while 66 applicants $(78.6 \%)$ secured preliminary spots. Of the preliminary residents, 37 (56\%) candidates eventually went on to secure categorical general surgery residency positions. The overall success rate based on gender was 69\% $(n=38)$ for the male applicants and $57 \%(n=17)$ for the female applicants. Median time to a categorical position was 3 (1-6) years after graduating from medical school. In our cohort $75 \%(n=41)$ of the graduates matched to a university program while $25 \%$ (n =14) to a community hospital program.

On average 5 graduates directly matched to or converted (from preliminary) to general surgery categorical positions every year while 6 graduates matched to preliminary positions annually. Figure 1 illustrates these numbers broken down by each respective year over a 10-year period (2008-2017). For comparison, Figure 2 illustrates the number of non-US IMGs matching into categorical positions and preliminary positions via the NRMP Match during the same 10-year period. Medical

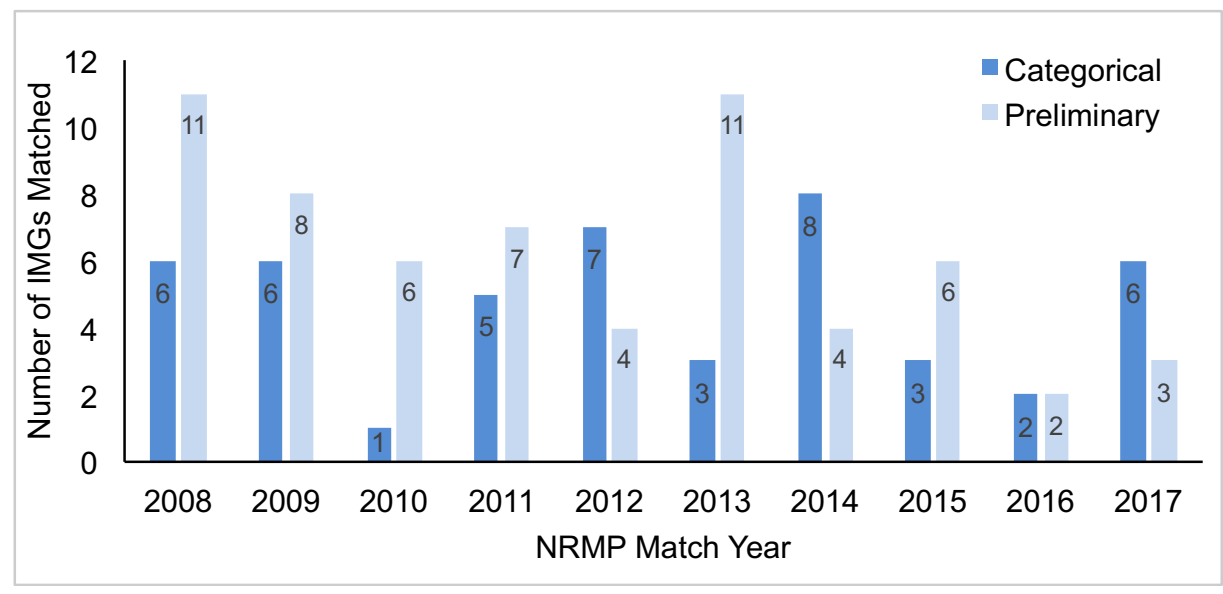

FIGURE 1. Number of medical graduates from Aga Khan University, Medical College (Karachi, Pakistan) matching into general surgery residency categorical and preliminary positions via National Resident Matching Program from 2008-2017.

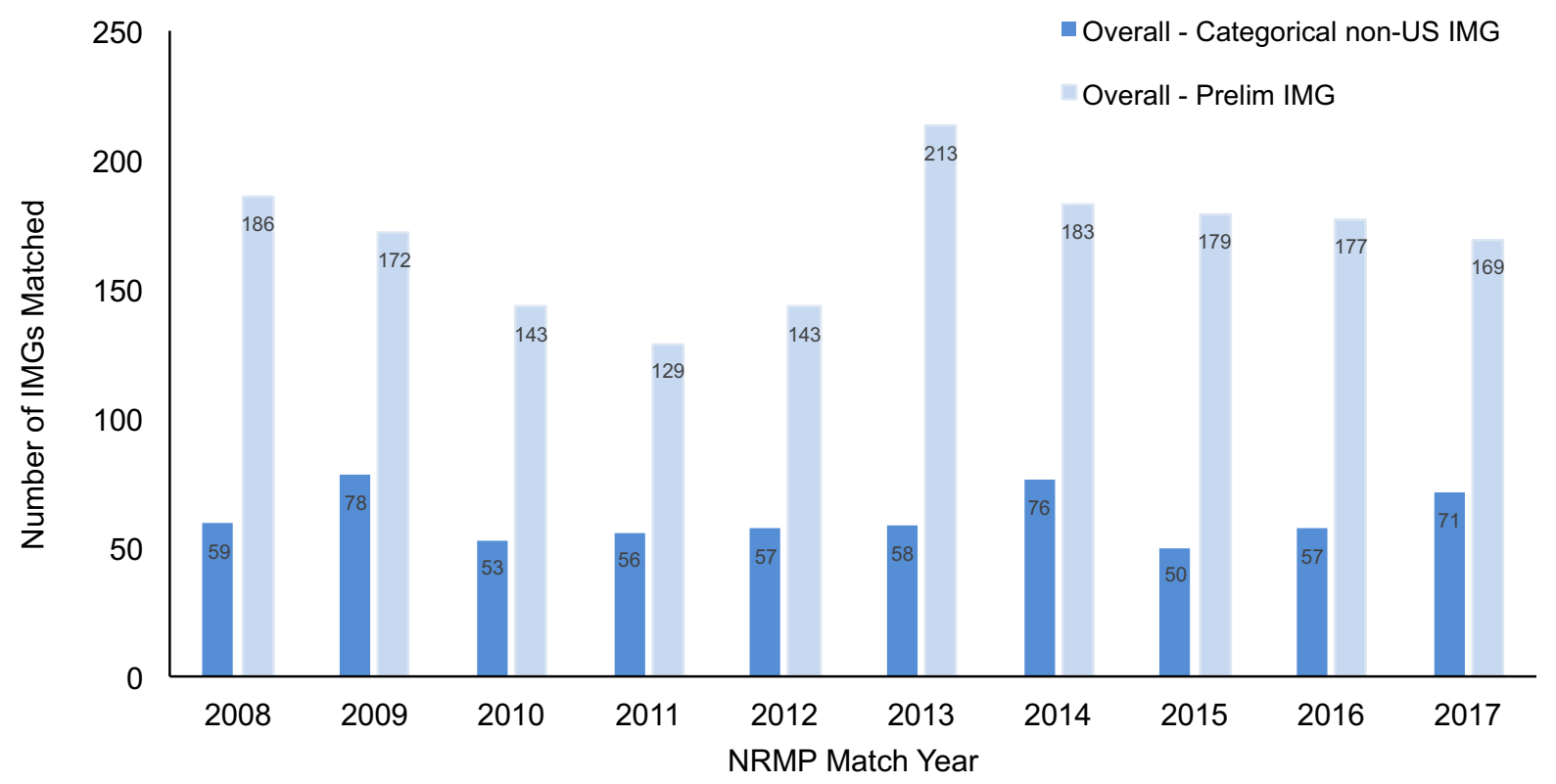

FIGURE 2. Number of non-US IMGs matching into general surgery categorical and preliminary positions via National Resident Matching ProgramP from 2008-2017. 

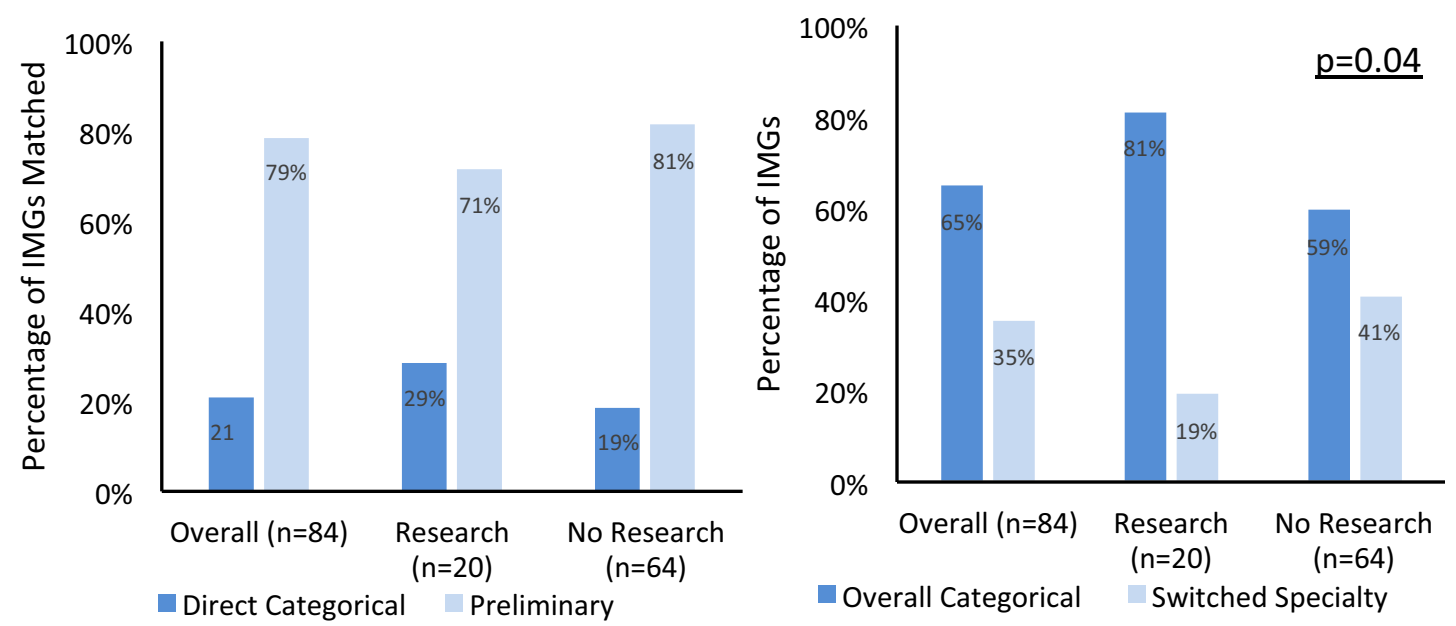

FIGURE 3. (a) Percentage of international medical graduates securing categorical positions compared to those matching into preliminary spots. (b) Proportion of residents eventually securing a categorical general surgery residency position compared to those switching specialties after starting out as preliminary general surgery residents.

graduates from AKU constitute 9\% of all non-US IMGs who have obtained categorical surgical residency positions in the last decade (Figure 3).

Upon analysis of our quasi-experimental groups: research group $(n=20)$ had an overall $85 \%(n=17)$ success rate of eventually matching into categorical positions. In the no-research group $(n=64)$, there was a 59\% $(\mathrm{n}=39)$ success rate (chi-square test, $\mathrm{p}=0.04)$ Preliminary to categorical conversion rates were $79 \%$ (Figure $3 \mathrm{~b}$ ). in the research group and $50 \%$ in the noresearch group. The research group had a higher chance of obtaining an eventual categorical spot (odds ratio 3.7, $\mathrm{p}=0.07$ ) or converting from a preliminary position to a categorical position (odds ratio $3.6, \mathrm{p}=0.06$ ), though neither was statistically significant. Our study is underpowered to detect this difference. For the research cohort the median time to obtaining a categorical position was 4 years (2-6), the median time for the noresearch group was 3 years (1-6). In the research group $82 \%(n=14)$ of the eventual categorical positions were at a university programs, while this number was $71 \%$ $(n=27)$ for the no-research group.

\section{DISCUSSION}

General surgery residency remains extremely competitive with match rates of $70 \%$ for graduates of US medical schools. Many IMGs choose to invest time in dedicated research positions before applications. The impact of such research on eventual outcomes remains largely unknown. The preference for research or direct application in this cohort of medical graduates was borne out of applicants' perceived perceptions of factors that they identified with higher chances of obtaining a surgical residency. Though we do not have objective board score data to stratify if the applicants' preference for research could be attributable to low board scores, we do have personal communications with this alumni network to suggest that this is not the case.

Our analysis of AKU Match list data for surgical applicants from 2008-2017 demonstrated a higher match rate in preliminary as compared to categorical general surgery residency positions. This finding is in congruence with the trends amongst IMGs seen across the world, with multiple US programs preferring to utilize a preliminary year as "trial" period before offering a categorical position, if available.

Our quasi-experimental study did demonstrate a higher success rate for applicants who had a significant research background in the US compared to those who did not. This finding has to date not been well supported in published literature. A study by Melendez et al. ${ }^{23} \mathrm{dem}-$ onstrated basic science and clinical research to be an important but secondary criterion for resident selection by general surgery residency program directors, with United States Medical Licensing Examination Step 1 scores and interviews being primary determinants. Makdisi et al. ${ }^{24}$ further showed prior research experience and publications to be the least important screening factors amongst selection criteria for surgical residents. According to the NRMP survey results from 2016 "demonstrated involvement and interest in research" was considered by only $48 \%$ of program directors when selecting candidates for interview selection. ${ }^{25}$ The same survey ranked this metric as the 28th (of 39) most important factor for ranking an applicant. Interactions with faculty and house-staff during interview, 
interpersonal skills, feedback from current residents, and United States Medical Licensing Examination Step 1/Comprehensive Osteopathic Medical Licensing Examination of the United States Level 1 scores being the top 5 criteria in the same survey. It has been suggested that use of research time may help boost an application if an applicant has marginal scores. However, we are unable to substantiate or refute this statement using our cohort, given that we do not have access to all board score reports.

One factor for this seemingly higher success rate for the research-group could be better social integration of IMGs in the US, prior to Match application, resulting in better performance during interviews and the application process. The existing credentialing requirements do not prepare IMGs for challenges presented by the US health system. ${ }^{26}$ It is possible that an extended duration of time spent in the US prior to the Match may have helped these IMGs in overcoming cultural and linguistic barriers leading to overall better outcomes in the match process.

Additionally, candidates who did research and applied for the Match could possibly be perceived as being more qualified, especially if they were productive with multiple publications during this research time. While we were unable to get this data for all 20 applicants (due to similarity in names and inability to distinguish from other authors), verifying against publicly available PubMed records and Google Scholar Citations we identified that applicants in the research-group had a median of 6 (range 2-23) indexed publications at the time of application. If this is further subdivided into those who did primarily laboratory-based research $(n=13)$ and those that participated in outcomes/clinical research $(n=7)$, the median publication number is 5 (range 2-13) and 14 (range 2-23) respectively. These numbers do not include abstracts or presentations at national meetings. These numbers are much higher than the NRMP averages for matched general surgery applicants in any given year this decade. A similar search (again incomplete due to overlapping and similar names) for the no-research group yielded a median of 1 (range 1-8) publication at time of application.

Another less quoted factor could be the effect of enhanced mentorship in research, which may ultimately lead to better outcomes for these candidates. The Table lists academic centres where research was conducted by these applicants. There could be intangible benefits of associating with faculty at such places or gaining access to contacts which could be beneficial in the Match process.

We recognize that statistical significance does not necessarily mean that real-world preferences of IMGs will change. However, in this instance we are considering
TABLE. List of Academic Centers and Hospital Programs Where Medical Graduates from AKU Pursued Research Prior to General Surgery Residency Applications

\begin{tabular}{llc}
\hline \multicolumn{2}{l}{ Research Center - Location } & Number \\
\hline Johns Hopkins University & Baltimore, MD & 4 \\
BIDMC/Harvard University & Boston, MA & 2 \\
MGH/Harvard University & Boston, MA & 2 \\
Yale University & New Haven, CT & 2 \\
Albert Einstein Medical Center & Albany, NY & 1 \\
Columbia University & New York, NY & 1 \\
Emory University & Atlanta, GA & 1 \\
Mayo Clinic & Rochester, MN & 1 \\
Mount Sinai Health System & New York, NY & 1 \\
St Barnabas Medical Center & Livingston, NJ & 1 \\
University of Alberta & Edmonton, Alberta & 1 \\
University of North Carolina & Chapel Hill, NC & 1 \\
Vanderbilt University & Nashville, TN & 1 \\
Washington University & St. Lovis, MO & 1 \\
\hline
\end{tabular}

career trajectory that can be dramatically impacted and should give some pause for thought, as these applicants already face an uphill task. We also recognize that most graduates in our cohort were initially preliminary residents. Their performance during those 1-2 preliminary clinical years was likely a decisive factor in the eventual outcome, however we believe that research years may have played a role in that performance too. The social integration and overcoming of cultural/linguistic barriers in many instances could have helped these applicants too.

Another reason for program reluctance in IMG selection may be a perceived damage to program reputation secondary to a decreased recruitment of US medical graduates. Moore et al. reported that despite a perception of nearly equal skill and ability, more than $70 \%$ program directors indicated that they believed IMGs were discriminated against. Nearly $20 \%$ reported that they ranked a less-qualified US medical graduate higher than a more qualified IMG, while $22 \%$ reported that they had ranked a US medical graduate higher than an IMG to avoid a reduced compliment of US medical graduates. ${ }^{27}$

Even in the presence of these barriers, the match rate for IMGs has been steadily rising. From 2008-2017, the average match rate for IMGs (US and non-US) was 61 positions/year for categorical spots (6\%) and 87 positions/year for preliminary spots across the US. ${ }^{28}$ We hope that this article will provide some insight into and direction for future IMG general surgery applicants, as well as for program directors evaluating IMG applicants.

\section{CONCLUSION}

Applicants who invested time doing dedicated research in the United States before applying for general surgery 
Match had better success rate in terms of securing categorical general surgery residency spots compared to applicants who applied directly. Preliminary residents with a research background also had higher chances of converting to categorical positions compared to preliminary residents applying directly.

\section{REFERENCES}

1. Zerehi R. For the Health and Public Policy Committee of the American College of Physicians. Creating a New National Workforce for Internal Medicine. A Position Paper. American College of Physicians; 2006.

2. About ECFMG: overview. Educational Commission for Foreign Medical Graduates website. Available at: http://www.ecfmg.org/about/index.html. Accessed February 8, 2015.

3. Ranasinghe PD. International medical graduates in the US physician workforce. J Am Osteopathic Assoc. 2015;115:236-241.

4. Lamb MN, Farley DR. The Mayo Clinic-Rochester experience with IMGs as general surgery trainees. Surgery. 2006; 140:351-353.

5. Association of American Medical Colleges; 2016. Available at: https://www.aamc.org/download/ 358920/data/surgery-gen.pdf. Accessed March 31, 2017.

6. Whitcomb ME, Miller RS. Comparison of IMGdependent and non-IMG-dependent residencies in the national resident matching program. JAMA. 1995;274:696-699.

7. Horvath K, Coluccio G, Foy H, Pellegrini C. A program for successful integration of international medical graduates (IMGs) into U.S. surgical residency training. Curr Surg. 2004;61:492-498.

8. Are C, Stoddard H, Carpenter LA, O'Holleran B, Thompson JS. Trends in the match rate and composition of candidates matching into categorical general surgery residency positions in the United States. Am J Surg. 2017;213:187-194.

9. The National Residency Matching Program. Main Residency Match Data: Historical Reports [Internet]. Washington, DC: NRMP.

10. Terhune KP, Zaydfudim V, Abumrad NN. International medical graduates in general surgery: increasing needs, decreasing numbers. J Am College Surg. 2010;210:990-996.
11. Cohen JJ. The role and contributions of IMGs: a US perspective. Acad Med. 2006;81:S17-S21.

12. Aranha GV. The international medical graduate in US academic general surgery. Arch Surg. 1998;133: 130-133.

13. Howard DL, Bunch CD, Mundia WO, et al. Comparing United States versus international medical school graduate physicians who serve African-American and white elderly. Health Serv Res. 2006;41:2155-2181.

14. Cardenas LF, Naik ND, Pandian TK, Gas BL, Strubel S, Cadeliña R, Heller SF. et al. A Comparison of Objective Assessment Data for the United States and International Medical Graduates in a General Surgery Residency. J Surg Educ. 2017;74:e1-e7.

15. Horvath K, Pellegrini C. Selecting international medical graduates (IMGs) for training in US surgical residencies. Surgery. 2006;140:347-350.

16. Christein JD, Cook JK, Enger TM, Farley DR. Preliminary general surgery residents: indentured servitude or golden opportunity? Curr Surg. 2006;63:85-89.

17. Petizman SF, McKinley D, Curtis M, Burdick W, Whelan GP. International medical graduates' performance of tech- niques of physical examination, with a comparison of US citizens and non-US citizens. Acad Med. 2002;75:S115-S117.

18. Boulet JR, McKinley DW, Whelan GP, Van Zanten M, Hambleton RK. Clinical skills deficiencies among first-year residents: utility of the ECFMG clinical skills assessment. Acad Med. 2002;77:S33-S35.

19. Schenarts PJ, Love KM, Agle SC, Haisch CE. Comparison of surgical residency applicants from US medical schools with US-born and foreign-born international medical school graduates. J Surg Educ. 2008;65:406-412.

20. Cockx B, Brasseur C. The demand for physician services. Evidence from a natural experiment. J Health Econ. 2003;22:881-913.

21. Bor J, Moscoe E, Mutevedzi P, Newell ML, Bärnighausen T. Regression discontinuity designs in epidemiology: causal inference without randomized trials. Epidemiology. 2014;25:729-737.

22. https://akuaana.org/resources/

23. Melendez MM, Xu $X$, Sexton TR, Shapiro MJ, Mohan EP. The importance of basic science and clinical research as a selection criterion for general surgery residency programs. J Surg Educ. 2008;65:151-154. 
24. Makdisi G, Takeuchi T, Rodriguez $J$, Rucinski J, Wise L. How we select our residents-a survey of selection criteria in general surgery residents. J Surg Educ. 2011;68:67-72.

25. http://www.nrmp.org/wp-content/uploads/2016/ 09/NRMP-2016-Program-Director-Survey.pdf

26. Meghani SH, Rajput V. Perspective: the need for practice socialization of international medical graduates-an exemplar from pain medicine. Acad Med. 2011;86:571-574.

27. Moore RA, Rhodenbaugh EJ. The unkindest cut of all: are international medical school graduates subjected to discrimination by general surgery residency programs? Curr Surg. 2002;59:228-236.

28. http://www.nrmp.org/main-residency-match-data/ 